\title{
Development of a database on radioresistant microorganisms as a useful tool for biotechnology
}

\author{
Alina Ryabova \\ KFU, Kazan, Russia \\ urban-nomad@yandex.ru
}

\author{
Azat Kadirov \\ KFU, Kazan, Russia \\ bilinet@mail.ru
}

\author{
Elena Shagimardanova \\ KFU, Kazan, Russia \\ NSU, Novosibirsk, Russia \\ rjuka@mail.ru
}

\begin{abstract}
Mechanisms of prokaryotic radioresistance are an essential subject in radiation biology. We designed a specialized database aimed at making a comprehensive repository of identified radioresistant microorganisms with experimentally confirmed data.
\end{abstract}

Keyword s-radioresistance, database, microorganism

Motivation and Aim

\section{Motivation}

We are yet far from detailed understanding of the extraordinary radioresistance strategies evolved by radioresistant organisms. There is an increasing need to compile the entire data about radioresistant microorganisms in a centralized and organized manner for better understanding the mechanisms of resistance to high doses of ionizing radiation.

Aim

We aim to collect and to make available for the scientific community the most up-to-date and relevant information about known radiation-resistant creatures. We designed the first version of database of radioresistant prokaryotes, as a preliminary step towards the development of a comprehensive resource for research and biotechnological purposes.

\section{Methods}

Based on the available literature, we selected studies from peer-reviewed journals supported by robust experimental data. As a result, about 80 species of radioresistant prokaryotes were selected, and their description formed the body of the database.

\section{Results}

The database schema consists of six tables, allowing to search and to retrieve any stored biological data. Among the main tables is the Taxonomy table with a detailed description of the systematic position of each species. The Irradiation table contains information about the type of ionizing radiation that the species has been exposed to, available experimental D10 values and detected lethal doses. The Mechanisms of Resistance table stores confirmed molecular mechanisms contributing to irradiation endurance. The next table refers to cross-resistance to other types of abiotic stresses. The Genomes table was obtained from NCBI databases, and contains data of sequenced strains for each species with NCBI taxonomy ID numbers, assembly level, total genome length $(\mathrm{Mb})$, percentage of GC content, gene and protein count, and accession numbers for GenBank and RefSeq assemblies. The later table provides a list of references. The species name is a central link for all tables in the database. This database of radioresistant microorganisms enables quick access and easy search for pertinent information with emphasis on irradiation procedures, mechanisms of protection and repair, and genome characteristics. We plan to expand the database, adding new species of radioresistant bacteria and archaea species, and to improve by integrating new tools into the database. Such a comprehensive repository could provide opportunities in biotechnology, bioengineering, or therapeutics for multiple diseases.

\section{ACKNOWLEDGMENT}

Supported by the Ministry of Science and Higher Education of the Russian Federation (id number: RFMEFI61419X0004). 